\title{
Characterization of the mass-dependent transmission efficiency of a CIMS
}

\author{
Martin Heinritzi ${ }^{1}$, Mario Simon ${ }^{1}$, Gerhard Steiner ${ }^{2,3}$, Andrea C. Wagner ${ }^{1}$, Andreas Kürten ${ }^{1}$, Armin Hansel ${ }^{3,4}$, and \\ Joachim Curtius ${ }^{1}$ \\ ${ }^{1}$ Goethe University Frankfurt, Institute for Atmospheric and Environmental Sciences, \\ 60438 Frankfurt am Main, Germany \\ ${ }^{2}$ University of Vienna, Faculty of Physics, 1090 Wien, Austria \\ ${ }^{3}$ University of Innsbruck, Institute for Ion and Applied Physics, 6020 Innsbruck, Austria \\ ${ }^{4}$ Ionicon Analytik GmbH, 6020 Innsbruck, Austria \\ Correspondence to: Martin Heinritzi (heinritzi@iau.uni-frankfurt.de)
}

Received: 21 August 2015 - Published in Atmos. Meas. Tech. Discuss.: 2 November 2015

Revised: 5 March 2016 - Accepted: 12 March 2016 - Published: 4 April 2016

\begin{abstract}
Knowledge about mass discrimination effects in a chemical ionization mass spectrometer (CIMS) is crucial for quantifying, e.g., the recently discovered extremely low volatile organic compounds (ELVOCs) and other compounds for which no calibration standard exists so far. Here, we present a simple way of estimating mass discrimination effects of a nitrate-based chemical ionization atmospheric pressure interface time-of-flight (CI-APi-TOF) mass spectrometer. Characterization of the mass discrimination is achieved by adding different perfluorinated acids to the mass spectrometer in amounts sufficient to deplete the primary ions significantly. The relative transmission efficiency can then be determined by comparing the decrease of signals from the primary ions and the increase of signals from the perfluorinated acids at higher masses. This method is in use already for PTR-MS; however, its application to a CI-APi-TOF brings additional difficulties, namely clustering and fragmentation of the measured compounds, which can be treated with statistical analysis of the measured data, leading to selfconsistent results. We also compare this method to a transmission estimation obtained with a setup using an electrospray ion source, a high-resolution differential mobility analyzer and an electrometer, which estimates the transmission of the instrument without the CI source. Both methods give different transmission curves, indicating non-negligible mass discrimination effects of the CI source. The absolute transmission of the instrument without the CI source was estimated with the HR-DMA method to plateau between the $m / z$
\end{abstract}

range of 127 and $568 \mathrm{Th}$ at around $1.5 \%$; however, for the CI source included, the depletion method showed a steady increase in relative transmission efficiency from the $m / z$ range of the primary ion (mainly at $62 \mathrm{Th}$ ) to around $550 \mathrm{Th}$ by a factor of around 5. The main advantages of the depletion method are that the instrument is used in the same operation mode as during standard measurements and no knowledge of the absolute amount of the measured substance is necessary, which results in a simple setup.

\section{Introduction}

The nitrate-based chemical ionization atmospheric pressure interface time-of-flight (CI-APi-TOF) mass spectrometer has been established as an important tool for atmospheric sciences over the last years. It is capable of measuring sulfuric acid down to levels of $5 \times 10^{4} \mathrm{~cm}^{-3}$ (Kürten et al., 2011; Jokinen et al., 2012) and clusters of sulfuric acid with dimethylamine (Kürten et al., 2014), as well as the newly described class of extremely low volatile organic compounds (ELVOCs, Ehn et al., 2014), which play a role in the formation of secondary organic aerosol (SOA). However, proper quantification of the measured signals remains challenging, as direct calibration gas standards for sulfuric acid, as well as for ELVOCs do not exist so far. For comparison, for protontransfer-reaction mass spectrometry (PTR-MS), which is capable of measuring precursors for ELVOCs like $\alpha$-pinene at 
the ppt level (Graus et al., 2010), calibration gas standards for many volatile organic compounds (VOCs) are commercially available with a typical uncertainty of $\pm 5 \%$. The lack of calibration gas standards for the CI-APi-TOF led to the development of a dedicated calibration system for sulfuric acid (Kürten et al., 2012). Here, a known amount of gaseous sulfuric acid is produced via UV-light induced generation of $\mathrm{OH} \cdot$ radicals (Tanner and Eisele, 1995) and subsequent oxidation of $\mathrm{SO}_{2}$ to $\mathrm{H}_{2} \mathrm{SO}_{4}$ (Eisele and Tanner, 1991). The UV system is characterized with a separate experiment, using photolytical conversion of $\mathrm{N}_{2} \mathrm{O}$ to $\mathrm{NO}_{x}$ (Edwards et al., 2003), where both reactant and product can be measured. With this system calibration factors for the CI-APi-TOF can be obtained for sulfuric acid with an overall uncertainty of around $33 \%$. However, this leaves still open the question of quantifying ELVOCs properly. Ehn et al. (2014) have performed a gravimetrical calibration of the CI-APi-TOF using perfluorinated heptanoic acid, which appears mainly in the $\mathrm{m} / z$ range of 319 to $426 \mathrm{Th}$ (monomers) and at $727 \mathrm{Th}$ (dimers). They obtained a similar calibration factor for this compound as for sulfuric acid; however, the detailed transmission efficiency of the instrument over the entire mass range cannot be resolved with this method.

The theoretical framework for deriving concentrations of a certain compound from ion count rates for the CI-APi-TOF is essentially the same as given by Eisele and Tanner (1993) for sulfuric acid measurements or by Hansel et al. (1995) for a PTR-MS. Ionization of a compound $\mathrm{X}$ via primary ions $\mathrm{P}^{ \pm}$ can be described by the following reaction:

$\mathrm{P}^{ \pm}+\mathrm{X} \stackrel{k}{\longrightarrow} \mathrm{X}^{ \pm}+\mathrm{P}$

The relation between primary ion concentration $\left[\mathrm{P}^{ \pm}\right]$and the concentration of the desired compound $[\mathrm{X}]$ in the reaction tube of the instrument is given by the following expression, where $k$ is the reaction rate constant for the ionization reaction:

$\frac{\mathrm{d}\left[\mathrm{P}^{ \pm}\right]}{\mathrm{d} t}=-k\left[\mathrm{P}^{ \pm}\right][\mathrm{X}]$.

Assuming $[\mathrm{X}]$ as constant over the reaction time $t$ (pseudo first order reaction approximation) enables a simple integration. The additional assumption that the total amount of charge is constant in the reaction tube leads to the following expression for the concentration of $[\mathrm{X}]$ :

$[X]=\frac{1}{k t} \ln \left(1+\frac{\left[\mathrm{X}^{ \pm}\right]}{\left[\mathrm{P}^{ \pm}\right]}\right) \approx \frac{1}{k t} \cdot \frac{\left[\mathrm{X}^{ \pm}\right]}{\left[\mathrm{P}^{ \pm}\right]}$.

Assuming $\left[\mathrm{X}^{ \pm}\right] \ll\left[\mathrm{P}^{ \pm}\right]$, which is usually fulfilled, allows for the first order approximation in Eq. (2). The concentration of compound $\mathrm{X}$ is proportional to the ratio of concentrations of product ions $\left[\mathrm{X}^{ \pm}\right]$and primary ions $\left[\mathrm{P}^{ \pm}\right]$. However, the mass spectrometer does not measure the ion concentrations in the reaction chamber directly, but only ion count rates at the location of the detector of the instrument. Therefore replacing the concentrations $\left[\mathrm{X}^{ \pm}\right]$and $\left[\mathrm{P}^{ \pm}\right]$in Eq. (2) by ion count rates $i\left(\mathrm{X}^{ \pm}\right)$and $i\left(\mathrm{P}^{ \pm}\right)$is only justified if we account for the relative mass discrimination of the two ion species inside the mass spectrometer:

$[\mathrm{X}] \approx \frac{1}{k t} \cdot \frac{i\left(\mathrm{X}^{ \pm}\right)}{i\left(\mathrm{P}^{ \pm}\right)} \cdot \frac{1}{T_{\mathrm{X}}}$.

Here, $T_{\mathrm{X}}$ is the factor that describes the mass discrimination of ions $\mathrm{X}^{ \pm}$relative to that of the primary ions $\mathrm{P}^{ \pm}$. This factor can be strongly dependent on the $m / z$ ratio of the involved ions. Calibration with an external gas standard or the above described $\mathrm{H}_{2} \mathrm{SO}_{4}$ calibration intrinsically accounts for this factor. However, if a direct calibration is not available, the factor $T_{\mathrm{X}}$ has to be known for the $m / z$ range of the desired compound. Therefore the calculated concentration of $\mathrm{X}$ depends on the ratio of product ions to primary ions, as well as the reaction time $t$, the reaction rate constant $k$ and the relative transmission factor $T_{\mathrm{X}}$. Ehn et al. (2014) showed with quantum chemical calculations that the collision rates of characteristic ELVOC molecules with the nitrate primary ions $\left(\mathrm{NO}_{3}^{-}\left(\mathrm{HNO}_{3}\right)_{0-2}\right)$ are equal to the collision rate of sulfuric acid with the nitrate primary ions, respectively. Furthermore, due to the assumed high binding energy between the highly oxidized ELVOC molecules and the nitrate primary ions, a charging at the kinetic limit is assumed, resulting in approximately the same reaction rate constant for ELVOCs as for sulfuric acid. This is, however, a rough approximation as the chemical composition of ELVOC molecules differs strongly and may thus result in varying reaction rate constants. As the reaction time is assumed to be equal for all compounds, only depending on the geometry of the ion source and the applied flows, the factor $1 / k t$ in Eq. (3) for ELVOC molecules would be approximately the same as for sulfuric acid, leaving the mass discrimination factor $T_{\mathrm{X}}$ as the only remaining unknown. This factor is of great importance for quantification of any uncalibrated compound and especially for ELVOC molecules, as their $\mathrm{m} / \mathrm{z}$ range (peaks between 235 and $625 \mathrm{Th}$ ) differs strongly from that of sulfuric acid (main peak at $97 \mathrm{Th}$ ).

In this study we show the application of the method of depleting primary ions to the nitrate-based CI-APi-TOF for obtaining transmission curves. We describe the measurement setup and the used compounds as well as the statistical analysis that became necessary due to the high level of clustering of compounds inside the CI-APi-TOF. Additionally we compare this method with a second approach for transmission estimations that uses a high-resolution differential mobility analyzer and an electrometer.

\section{Mass discrimination effects}

Various parts of the time-of-flight mass spectrometer (Tofwerk AG, Thun, Switzerland) can cause mass discrimination 
effects, especially the two quadrupole units in the APi interface, the orthogonal extraction unit in the TOF and the multichannel plate (MCP) detector. The CI source itself (Kürten et al., 2011) may also cause mass discrimination effects, as several negative voltages are applied to the source to guide the ions to the pinhole, which marks the entrance of the vacuum region of the instrument. After the pinhole, three differentially pumped chambers are installed, two of which contain a segmented quadrupole operating in RF-only mode, and the last one contains an Einzel lens system. The main task of these transfer units is to guide and focus the ion beam to the high vacuum TOF region. Quadrupoles in RF-only mode act as a high pass filter for ions as the trajectories of ions below a certain $\mathrm{m} / \mathrm{z}$ ratio are not stable any more (Chernushevich et al., 2001). On the other hand, as the potential well provided by a quadrupole is inversely proportional to $m / z$, heavier ions suffer poorer focusing and thus reduced transmission (Gerlich, 1992). The $m / z$ range with maximum transmission can however be modulated with the applied RF-voltage. According to Chernushevich et al. (2001), higher RF voltages lead to a stronger suppression of lighter ions and vice versa. Additionally, the shape of the transmission curve depends on the ion beam shape at the entrance of the quadrupole. Muntean (1995) showed ion trajectory calculations taking into account ion beam divergence as well as off-axis beam profiles. While beam divergence shifts the maximum transmission to small values, meaning an additional suppression of heavy ions, off-axis intensities have the opposite effect. In practice, there will be a suppression of ion transmission at both ends of the $m / z$ range, with the shape and position of the transmission window depending on the detailed settings and the quality of the beam focusing.

The orthogonal extraction unit of a TOF shows a systematic discrimination of lower $m / z$ ratios under the assumption of a mono-energetic ion beam entering the extraction unit in axial direction (Chernushevich et al., 2001). This discrimination is a result of the higher axial velocity and thus larger spatial spread of lighter ions along the axis. Therefore as a larger fraction of lighter ions has already left the extraction region compared to heavier ions, fewer of the lighter ions are accelerated orthogonally to the detector. As the velocity of the ions is proportional to $\sqrt{m / z}^{-1}$ the geometric duty cycle is proportional to $\sqrt{m / z}$.

Also the multi-channel plate detector in the TOF can show mass discrimination effects. Müller et al. (2014) showed for a PTR-TOF, which utilizes the same MCP as the TOF in this study (PHOTONIS Inc., Sturbridge, MA, USA), that the mass discrimination can change due to aging of the detector and is strongly dependent on the operating voltage of the MCP. Especially at low gain voltages, higher masses are discriminated against more strongly. Gilmore and Seah (2000) showed that the mass discrimination effects of an MCP also vary with the total impact energy of the ions, which is defined by the post accelerating voltage in a TOF. This study found a reduced detection efficiency for heavier ions at lower impact energies.

Finally, even the smallest changes of the inner geometry of the instrument, e.g., caused by shipping, can affect ion beam alignment and thus cause different transmission characteristics. This variety of effects makes it almost impossible to calculate the transmission efficiency, so methods for a measurement of this property are crucial.

\section{Methods}

\subsection{HR-DMA}

The development of high-resolution differential mobility analyzers (HR-DMA) led to the ability of classifying particles and clusters down to a size of around $1 \mathrm{~nm}$ (Steiner et al., 2010; Fernández de la Mora et al., 2013). As this corresponds to a mass range the CI-APi-TOF is able to measure, these two instruments can be combined for a characterization of the latter. Here, a version of the so called "Vienna-type" DMA was used (Steiner et al., 2010). It is a closed loop, medium flow (up to around $700 \mathrm{slpm}$ ) size classifier, with a maximum resolution power of around 20 . The resolution power is derived from the ratio of the electrical mobility $Z$ of a certain cluster and the full-width half maximum (FWHM) of the corresponding peak in a mobility scan. However, for the operating conditions in this study a resolution power between 10 and 15 was achieved, which was sufficient to clearly separate monomers and dimers of almost all compounds tested. The high resolution, in principle, is necessary to distinguish between small clusters with only one molecule difference in mass. To generate these clusters, an electrospray ion source was used, which can be operated in either negative or positive mode. As the nitrate-based CI-APi-TOF is operating in negative ion mode, we produced negatively charged clusters of several bromide and iodide salts as well as an ionic liquid (see Table 1). After production by electrospray ionization (ESI) and size selection in the HR-DMA the cluster ion flow is split up between a Faraday cup electrometer (EM) to measure the ion count rate in front of the mass spectrometer and the CI-APi-TOF. The transmission efficiency of the mass spectrometer at a certain $\mathrm{m} / \mathrm{z}$ ratio is obtained by dividing the ion count rates from the TOF by the corrected EM ion count rate. The configurations that were tested are shown in Fig. 1a and b. This method is in use already for characterizing the transmission efficiency of APi-TOF instruments (Junninen et al., 2010) and does not account for mass discrimination effects of the CI source. The pinhole plate of an APi-TOF is usually set to a certain voltage to attract ions. In the CI-APi-TOF this is achieved by applying negative voltages to the CI source, whereas the pinhole plate itself is at ground potential. In the configurations used here, the pinhole plate was at ground as well in order not to disturb the potential steps on the vacuum side of the instrument. In the 
Table 1. Compounds used for HR-DMA method with according monomer, dimer, and trimer. Trimers were not always quantifiable as the HR-DMA also selected multiply charged higher clusters when adjusted for trimers (indicated by italics). This was also the case for the tetradodecylammonium bromide dimer.

\begin{tabular}{llll}
\hline Name & Monomer, $m / z[\mathrm{Th}]$ & Dimer, $m / z[\mathrm{Th}]$ & Trimer, $m / z[\mathrm{Th}]$ \\
\hline $\begin{array}{l}\text { Methyltrioctylammonium } \\
\text { bis(trifluoromethylsulfonyl)imide, MTOA }\end{array}$ & $\mathrm{C}_{2} \mathrm{NO}_{4} \mathrm{~F}_{6} \mathrm{~S}_{2}^{-}, 279.92$ & $\mathrm{C}_{29} \mathrm{H}_{54} \mathrm{~N}_{3} \mathrm{O}_{8} \mathrm{~F}_{12} \mathrm{~S}_{4}^{-}, 928.26$ & $\mathrm{C}_{56} \mathrm{H}_{108} \mathrm{~N}_{5} \mathrm{O}_{12} \mathrm{~F}_{18} \mathrm{~S}_{6}^{-}, 1576.60$ \\
Tetrabutylammonium ioidide, TBAI & $\mathrm{I}^{-}, 126.90$ & $\mathrm{C}_{16} \mathrm{H}_{36} \mathrm{NI}_{2}^{-}, 496.09$ & \\
Tetrapropylammonium iodide, TPPAI & $\mathrm{I}^{-}, 126.90$ & $\mathrm{C}_{12} \mathrm{H}_{28} \mathrm{NI}_{2}^{-}, 440.03$ & \\
Tetraheptylammonium bormide, THABr & $\mathrm{Br}^{-}, 78.92$ & $\mathrm{C}_{28} \mathrm{H}_{60} \mathrm{NBr}_{2}^{-}, 568.31$ & \\
Tetradodecylammonium bromide, TDABr & $\mathrm{Br}^{-}, 78.92$ & $\mathrm{C}_{48} \mathrm{H}_{100} \mathrm{NBr}_{2}^{-}, 848.62$ & \\
\hline
\end{tabular}

first setup the cluster ions are directly guided to the pinhole of the TOF, which marks the entrance to the vacuum region. The goal of this setup was to determine the mass discrimination of the TOF itself, including the APi section from the point of the pinhole to the detector (sampling line effects are discussed below). However, we also chose a second setup, where the CI source of the instrument was installed, with the goal of studying possible effects from this section on the transmission curve. All the voltages of the CI source were set to standard operation values, as well as all flows. The corona discharge was shut off and nitric acid was not fed into the instrument. Choosing this setup, one faces the problem that the CI source is operated at negative voltages up to $-500 \mathrm{~V}$, so feeding negative ions from outside into the source means climbing a very large potential step. This step can of course affect ions with different masses in different ways; we expect high losses of light ions due to their high electrical mobility. Instead of placing this potential step at the end of the $1 / 2^{\prime \prime}$ sampling line inside the CI source, we chose to set it outside the ion source, where we installed a $6 \mathrm{~mm}$ nylon insulator (Fig. 1b). Due to the smaller inner diameter, the velocity of the flow is higher which should give smaller losses of ions at the voltage step. However, this way the usually grounded sampling line as well as the first part of the ion source had to be set to $-500 \mathrm{~V}$.

Measuring only in the configurations given in Fig.1a and b yields the total transmission curve of the whole setup including tubing effects. For technical reasons the sampling line to the TOF did not have the same length as the line to the EM, therefore the losses of ions in the lines cannot be assumed to be identical. Additionally, the sampling line of the TOF increases in diameter from $6 \mathrm{~mm}$ to $1 / 2^{\prime \prime}$ in front of the CI source in the second setup. It is crucial to correct for these losses, as diffusion to the walls is mass-dependent and thus affects the shape of the transmission curve. We have made additional measurements to obtain mass-dependent EM correction factors that account for the different tubing lengths and flows (see Fig. 2). By doing so, we can estimate the ion concentration not only at the position of the EM, but directly in front of the mass spectrometer which is the important measure for the transmission estimation. For that we

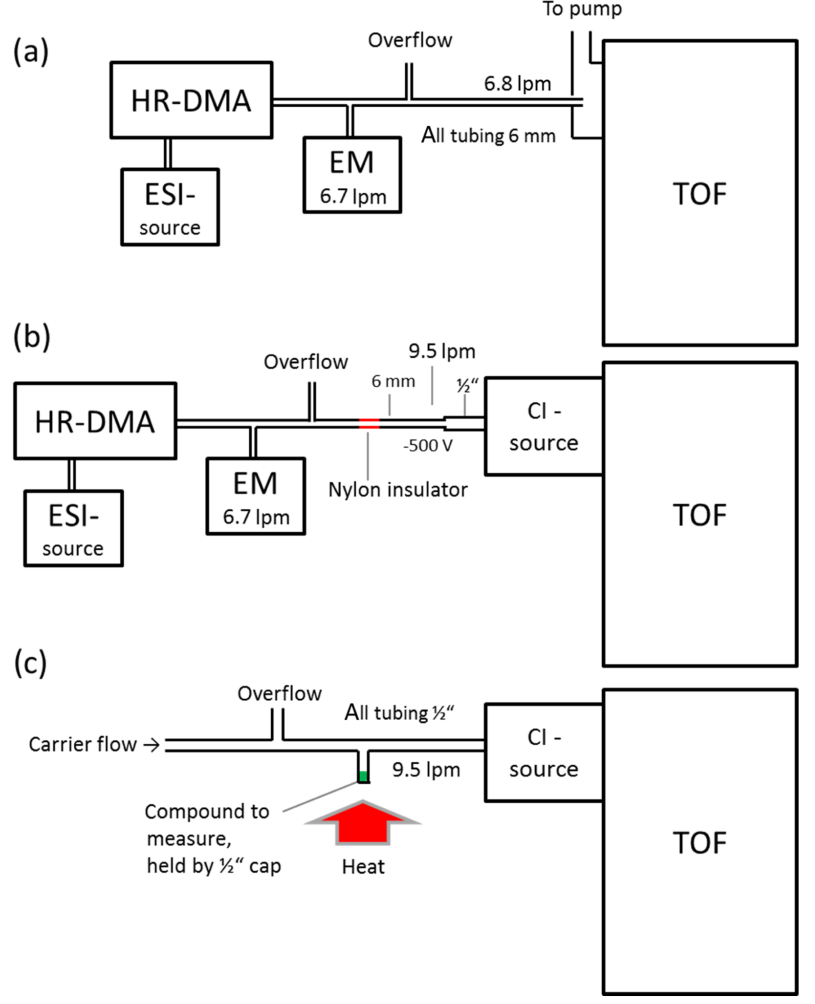

Figure 1. Schematic setup for the three types of experiments conducted. (a) HR-DMA measurement without CI source, APi-TOF only. (b) HR-DMA setup with CI ion source. The $1 / 2^{\prime \prime}$ sampling line of the CI source was at a potential of $-500 \mathrm{~V}$. To minimize losses the potential step from ground to $-500 \mathrm{~V}$ was installed at the $6 \mathrm{~mm}$ line with a nylon insulator. (c) Setup for the depletion method. A small sample was stored in a $1 / 2^{\prime \prime}$ cap and slowly heated with a standard heat gun. Potential overly high or overly small diffusion of the sample into the carrier flow can be controlled by varying length and inner diameter of the vertical connection between the sample and the carrier flow line.

placed the EM at the position of the TOF and applied equal flows as during the standard measurements. However, as the inlet of the EM has $6 \mathrm{~mm}$ diameter, the transition from $6 \mathrm{~mm}$ to the $1 / 2^{\prime \prime}$ TOF sampling line could not be accounted for. 
This is a remaining source of uncertainty as losses at this point may be mass-dependent as well. For the CI setup we also tried to simulate the potential step of $-500 \mathrm{~V}$ by using a nylon insulator; however, we used a second nylon insulator to set the sampling line to ground again in front of the EM. As only one EM was available the losses could not be measured simultaneously, but only after reassembling the setup, assuming the ESI source performance stayed constant over the duration of the measurement and reassembling. This was checked by measuring in the initial position of the EM before and after assembling, which showed reasonable agreement. The measurements were performed with the monomer, dimer, and trimer of MTOA (methyltrioctylammonium bis(trifluoromethylsulfonyl)imide), which correspond to $\mathrm{m} / \mathrm{z}$ ratios of $279.9,928.3$, and $1576.6 \mathrm{Th}$, respectively. The mobility spectra for these measurements are shown in Fig. 2a and b. Each mobility spectrum is the average of 5 individual scans. The ratios of the individual peak areas for EM at original position and at TOF position give the mass-dependent correction factors. Peak areas were obtained by fitting triple Gaussian peaks to the spectra. The MTOA trimer signal is strongly affected by multiply charged heavier clusters selected by the HR-DMA due to their identical electrical mobility. The shoulder in the data caused by these higher clusters was fitted with a double Gaussian peak and subtracted from the trimer signal before obtaining trimer peak areas. As these measurements were only performed for MTOA monomer, dimer, and trimer, covering a $\mathrm{m} / \mathrm{z}$ range from 280 to $1576 \mathrm{Th}$, we had to extrapolate the EM correction to smaller clusters (see Fig. 2c). For that an exponential fit with offset 1 was applied to the correction factors, whereas the MTOA trimer was only weighted with $50 \%$ due to the effects of multiply charged higher clusters. With this extrapolation, additional uncertainty is introduced to the EM correction of especially negative monomers of bromide and iodide salts $\left(\mathrm{Br}^{-}\right.$at $79 \mathrm{Th}$ and $\mathrm{I}^{-}$at $\left.127 \mathrm{Th}\right)$. In general, both setups are not perfect; as in the APi setup, the ions are not drawn to the pinhole, whereas in the CI setup a large potential step and thus large correction factors are involved. The total uncertainty consists of contributions from the EM correction for MTOA ( $8 \%$ ), the extrapolation of the fit to other masses (estimated by using $68 \%$ confidence bonds of the EM correction fit to be around $7 \%$ ), and for dimers possible fragmentation to monomers. Especially iodide dimers fragmented strongly to $\mathrm{I}^{-}$, the EM values were corrected for that with the independently obtained monomer transmission efficiency. The percentage of EM signal attributed to lower clusters was used to estimate the additional uncertainty introduced by fragmentation. As the MTOA trimer signal overlaps with signals from multiply charged heavier clusters and the maximum correction of the MTOA peak in the mobility spectrum due to the shoulder was $31 \%$, this number was used as additional uncertainty for the MTOA trimer. During the EM correction measurements for the CI mode a second nylon insulator was installed to operate the EM at ground potential.
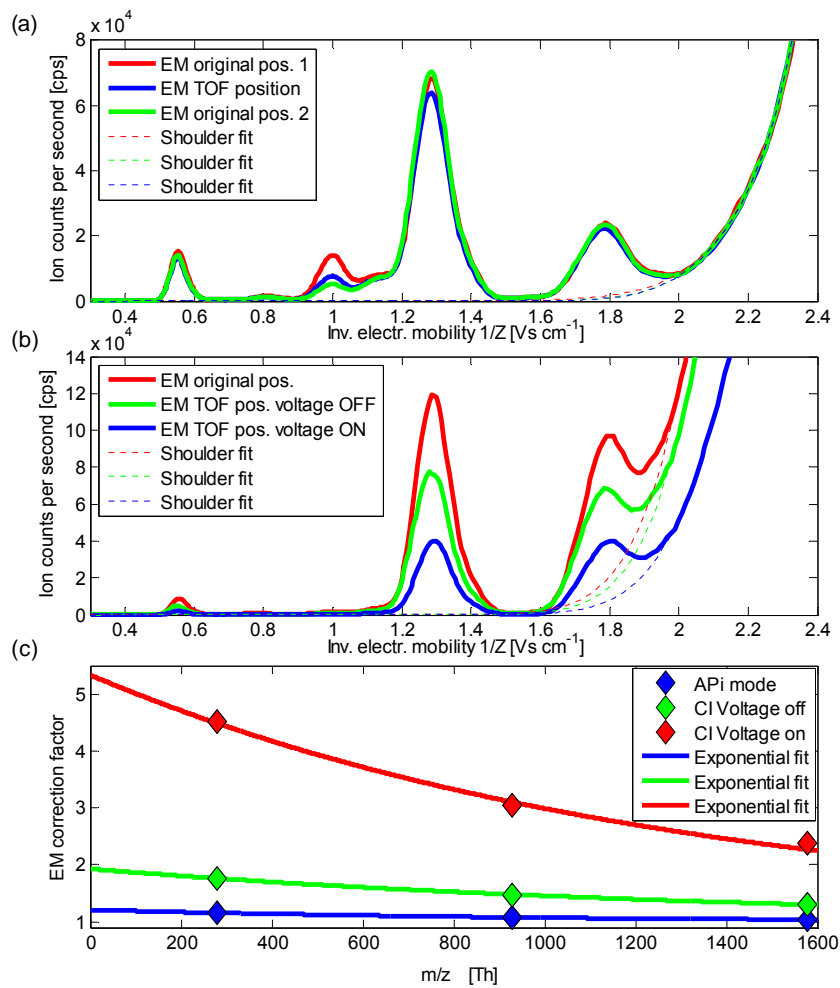

Figure 2. (a) Mobility spectrum for MTOA generated by the HRDMA with the EM at original position and when its position was interchanged with that of the APi-TOF. The shoulder on the right originates from multiply charged higher clusters. The fit of the shoulder to correct the trimer concentration is shown as well. (b) Same as (a) but with two nylon insulators and potential step of $-500 \mathrm{~V}$ added to the setup. Applying the voltage results in additional drastic losses. (c) Calculated correction factors for MTOA monomer, dimer, and trimer and exponential fit for extension to smaller $m / z$ ratios.

Ion losses in this second insulator were not quantifiable and are thus not represented by error bars, but we note that there may be additional uncertainty for the data points of the CI mode. This may lead to an underprediction of ion concentration in front of the pinhole and thus an overprediction of the CI mode transmission values.

\subsection{Depletion of primary ions}

The basic idea of the depletion method is to add different substances to the instrument one after the other in amounts that lead to a significant decrease in primary ions (Steinbacher et al., 2004; Huey et al., 1995). Charge provided by the primary ions is therefore shifted to the $\mathrm{m} / \mathrm{z}$ region of the product ions. Comparing the loss of charge at the primary ion mass with the appearance of charge at the product ion mass yields the relative transmission factor between these two masses. The underlying assumption is that the total amount of charge in the system is constant over the duration of the experiment, 
which is usually the case for both PTR-MS, as well as CIAPi-TOF. The substances should ideally not fragment significantly nor cluster with themselves as this leads to a charge transfer to a third mass region; however, we will face this problem for the CI-APi-TOF. The relation between reacted primary ions and product ions should be linear and the slope of the linear fit gives the relative transmission of the $m / z$ range of the product ions compared to the $m / z$ range of the primary ions. Adding different compounds one after the other leads to a transmission curve over a certain $m / z$ range. The first main advantage of this method is that it is not necessary to know how much of the compound of interest is added to the instrument. This leads to a very simple measurement setup without the need for further instrumentation. The second main advantage is that the instrument is used in standard operating mode including the CI source, which is not the case for the HR-DMA method. Therefore the depletion method intrinsically accounts for mass discrimination effects of the CI source. Note that this method only reveals the relative mass discrimination of the product ions compared to the $m / z$ range of the primary ions, which is however sufficient for quantification (Eq. 3). If the absolute transmission is needed, the HR-DMA method should be chosen.

The saturation method works fine for a PTR-MS as this technique is a rather soft ionization method and there is almost no clustering of a substance with itself. Therefore the shift of charge from the $m / z$ range of the primary ions to the desired $m / z$ range is usually rather complete, and almost no charge appears at a third $\mathrm{m} / \mathrm{z}$ range; however, this may change for certain substances. Good compounds for applying this method to a PTR-MS are for example acetone or xylene, whereas monoterpenes usually show a considerable degree of fragmentation. The picture changes, however, when we want to apply this technique to the CI-APi-TOF. First of all, we need different substances that can be easily measured with the CI-APi-TOF and should cover substantial parts of the $\mathrm{m} / \mathrm{z}$ range (ideally from around 100 to around $1500 \mathrm{Th}$ ). We have chosen several perfluorinated acids $\left(\mathrm{CF}_{3}\left(\mathrm{CF}_{2}\right)_{3,5,6,7,8} \mathrm{COOH}\right.$, Sigma Aldrich, purity $\geq$ $95 \%$ ) as fluorine is very electronegative and is thus supposed to easily take over the negative charge from $\mathrm{NO}_{3}^{-}$, while the hydrogen atom of the acid group can perform a proton transfer reaction with the primary ion. On the other hand, perfluorinated acids are available at different molecular weights, with a variable number of $\mathrm{CF}_{2}$ building blocks included. $\mathrm{CF}_{2}$ has a molecular mass of $50 \mathrm{amu}$, so the $\mathrm{m} / \mathrm{z}$ range can be stepped through easily by adding differently sized compounds. We have tried also perfluorododecyltrichlorosilane $\left(\mathrm{C}_{12} \mathrm{H}_{4} \mathrm{Cl}_{3} \mathrm{~F}_{21} \mathrm{Si}\right.$, Sigma Aldrich, purity $\left.97 \%\right)$. Table 2 shows an overview of the substances that were applied.

The measurement setup has to ensure that sufficient amounts of the desired compound enter the CI source of the instrument to deplete the primary ions, but on the other hand one has to be careful not to put too much into the system to avoid long term contamination. One way to achieve this is to inject a gas sample from the headspace of the desired compound with a needle into the sample flow of the instrument through a rubber sealing. This works in principle, but gives only very short signal spikes as the volume in the needle is quickly diluted. A better approach is shown in Fig. 1c), where a $1 / 2^{\prime \prime}$ T-piece was added to the sampling line with a short $1 / 2^{\prime \prime}$ tube and a $1 / 2^{\prime \prime}$ cap at the end of the tube. The chosen compound is placed inside the cap. It is important to only use very small amounts in order not to contaminate the whole system for long periods of time. All the substances used evaporated either from liquid (perfluorinated pentanoic acid) or solid phase (all others) and diffused along the short vertical $1 / 2^{\prime \prime}$ line into the sample flow. Different concentration levels of the compounds were achieved by heating the cap slowly with a heat gun. If the amount of a compound in the cap is too large, a permanent complete depletion of all primary ions can occur. As will be described below, we need, however, varying degrees of depletion to deliver meaningful transmission curves; a complete and permanent depletion of the primary ions is not useful. To avoid this, it is important to only use a small grain/drop of the respective compound and/or to install a longer and/or thinner vertical diffusion tube in the inlet setup (see Fig. 1c).

The spectra for the different compounds are shown in Fig. 3. It is evident that the charge provided by the primary ions is not only shifted to one distinct $m / z$ region like it is the case for this method in PTR-MS, but distributed over a wider range of masses. The monomer of all perfluorinated acids appears at three main channels, namely proton transfer to $\mathrm{NO}_{3}^{-}$(middle peak), clustering with $\mathrm{NO}_{3}^{-}$(right peak) and fragmentation (loss of the acid group; left peak). These three monomer peaks alone span already over a $\mathrm{m} / \mathrm{z}$ range of $106 \mathrm{Th}$, which is a disadvantage of this method. Furthermore, the perfluorinated acids, as well as the silane compound tend to cluster strongly with themselves. However, for dimers and trimers of these acids, only one main peak was measured originating from a proton transfer reaction. Higher clusters like tetramers or pentamers were not observed. Especially for smaller perfluorinated acids, the tetramers would lie well below $2000 \mathrm{Th}$, which is the highest $\mathrm{m} / z$ ratio the CI-APiTOF is able to measure; so it is reasonable to assume that we are not missing any charge at $m / z$ ratios higher than $2000 \mathrm{Th}$. We also know from different experiments that the transmission efficiency for high $\mathrm{m} / z$ ratios of up to $2000 \mathrm{Th}$ is not zero.

To obtain time series of the data we used the MATLAB based tofTools software. For the monomer intensities of the perfluorinated acids and the silane compound, all monomer peaks were added up without any further correction for transmission. The measurement data from the heating experiments with perfluorinated heptanoic and nonanoic acid (Fig. 4) show a decrease of the primary ion signal (sum of $\mathrm{NO}_{3}^{-}$and $\mathrm{HNO}_{3} \mathrm{NO}_{3}^{-}$) when the heating started at the beginning of the experiment and a strong increase in the perfluorinated acid signal. The sum of all signals also increases by 
Table 2. Compounds used for depletion method. The perfluorinated acids only differ in the number of $\mathrm{CF}_{2}$ building blocks.

\begin{tabular}{llrr}
\hline Name & Formula & Mol. weight [amu] & Purity [\%] \\
\hline Perfluorinated pentanoic acid & $\mathrm{CF}_{3}\left(\mathrm{CF}_{2}\right)_{3} \mathrm{COOH}$ & 264.05 & 97 \\
Perfluorinated heptanoic acid & $\mathrm{CF}_{3}\left(\mathrm{CF}_{2}\right)_{5} \mathrm{CO}_{2} \mathrm{H}$ & 364.06 & 99 \\
Perfluorinated octanoic acid & $\mathrm{CF}_{3}\left(\mathrm{CF}_{2}\right)_{6} \mathrm{COOH}$ & 414.07 & 96 \\
Perfluorinated nonanoic acid & $\mathrm{CF}_{3}\left(\mathrm{CF}_{2}\right)_{7} \mathrm{COOH}$ & 464.08 & 97 \\
Perfluorinated decanoic acid & $\mathrm{CF}_{3}\left(\mathrm{CF}_{2}\right)_{8} \mathrm{CO}_{2} \mathrm{H}$ & 564.09 & 95 \\
Perfluorododecyltrichlorosilane & $\mathrm{C}_{12} \mathrm{H}_{4} \mathrm{Cl}_{3} \mathrm{~F}_{21} \mathrm{Si}$ & 681.57 & 97 \\
\hline
\end{tabular}

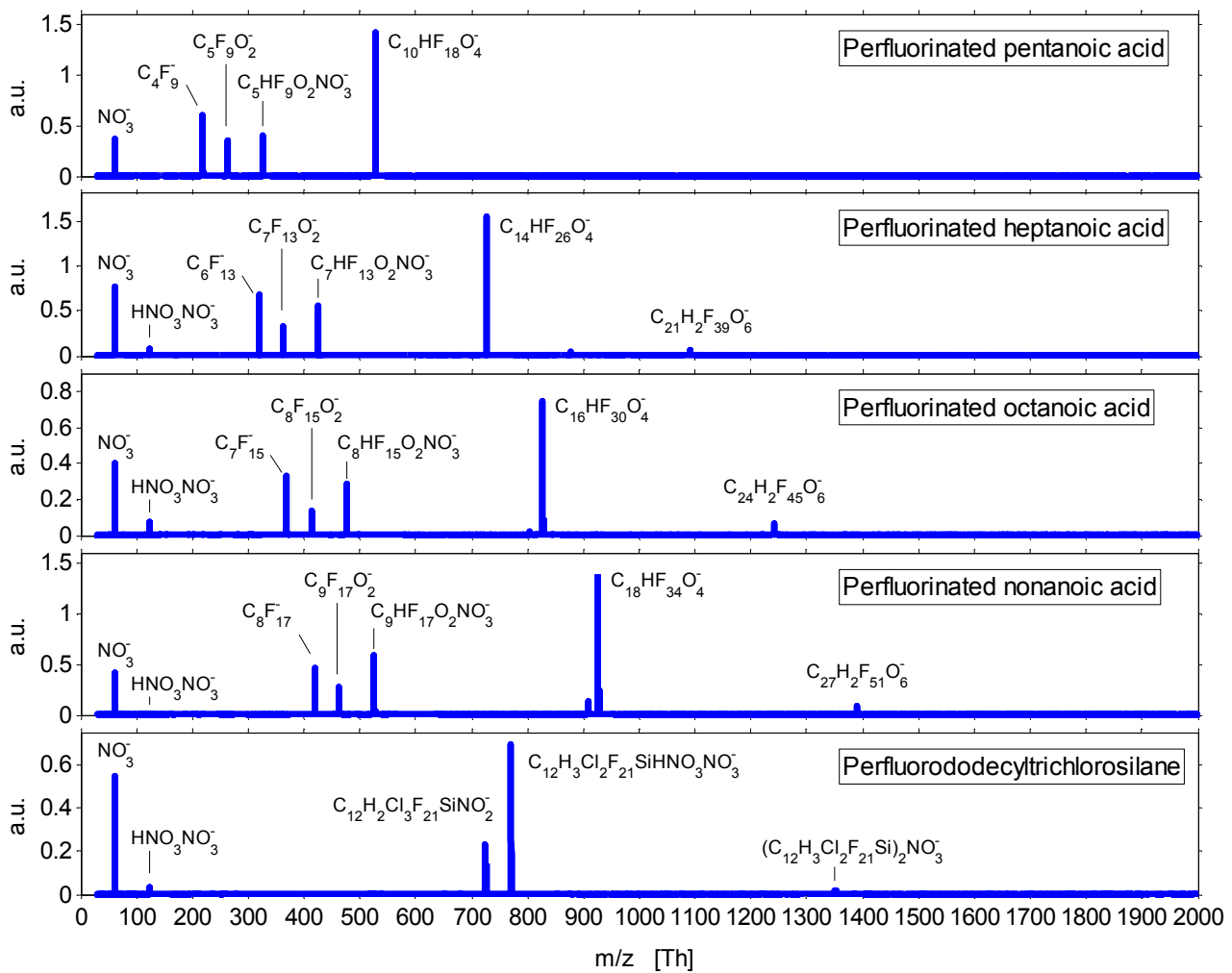

Figure 3. Spectra for all compounds used for depletion mode experiments. Primary ions are $\mathrm{NO}_{3}^{-}$at $62 \mathrm{Th}$ and $\mathrm{HNO}_{3} \mathrm{NO}_{3}^{-}$at $125 \mathrm{Th}$. The three monomer peaks for the perfluorinated acids originate from proton transfer to $\mathrm{NO}_{3}^{-}$(middle peak), clustering with $\mathrm{NO}_{3}^{-}$(right peak) and fragmentation (loss of the acid group, left peak). Dimers and trimers of the perfluorinated acids are present only as one main peak. Notice the stepwise addition of $\mathrm{CF}_{2}$ building blocks for the perfluorinated acids.

more than a factor of 2 . The total amount of charge in the CI source is given by the primary ion production in the corona discharge and is assumed to be constant over the experiment. As we count more charge during the heating at higher $\mathrm{m} / \mathrm{z}$ regimes, the transmission efficiency of charge through the instrument must be higher for the $m / z$ range of heptanoic and nonanoic acid monomer and dimer compared to the $m / z$ range of the primary ions.

However, as the charge from the primary ions is not only transferred to one distinct mass, but distributed over a wider range, we cannot apply a linear fit to the data. We therefore introduce a relation that combines the measured signal with the theoretical total amount of charge in the system via weighting factors, which will lead to relative transmission factors:

$$
\begin{aligned}
& s_{\text {prim }} \cdot f_{\text {prim }}+s_{\text {mon }} \cdot f_{\text {mon }}+s_{\text {dim }} \cdot f_{\text {dim }} \\
& \quad+s_{\text {trim }} \cdot f_{\text {trim }}=1
\end{aligned}
$$

or $\sum_{i} s_{i} f_{i}=1$, where $i$ indicates primary ions and monomers, dimer, and trimer of the perfluorinated acids. $s_{i}$ is the signal of the corresponding compound in counts per second at a given time and $f_{i}$ are the weighting factors that need to be introduced to yield the right hand side of the equation, which is a normalized expression of the total amount of charge present in the CI source. This is a quantity not directly measurable 

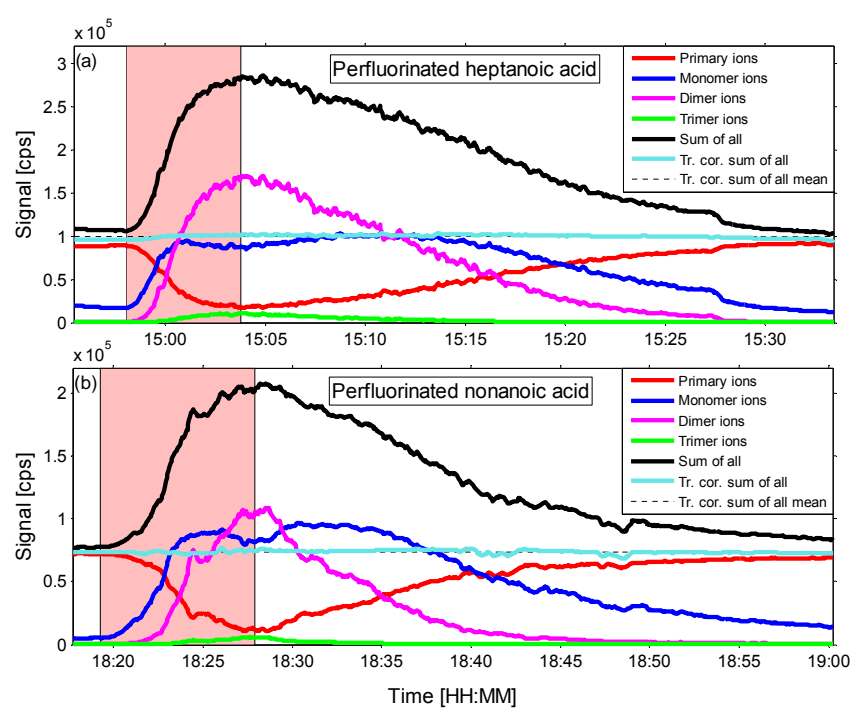

Figure 4. Measurement examples for (a) perfluorinated heptanoic acid and (b) perfluorinated nonanoic acid. Data are slightly smoothed (moving average). Heating (indicated by red shaded areas) leads to increased evaporation of the perfluorinated acids and a depletion of primary ions. After the heating period the system cooled down to room temperature, with the corresponding decrease in signal for perfluorinated acids and recovery of the primary ions. The sum of all signals shows a clear increase due to the heating periods, as charge is shifted to higher $m / z$ regions. As the amount of charge is limited by the primary ions, this indicates that a higher fraction of ions reaches the detector when the ions are at the $m / z$ range of the monomer or dimer of pentanoic or nonanoic acid. This is direct evidence for higher transmission efficiencies at these $\mathrm{m} / \mathrm{z}$ ranges.

with the TOF unless the absolute transmission efficiency is known. We are not interested in its absolute value, but just need to assume that it is constant over the duration of the experiment. Equation (4) has four knowns and four unknowns, but it must be true for every time step of the experiment, so this one equation expands into an overdetermined system of linear equations, which can readily be solved via regression analysis. After obtaining the four weighting factors $f_{i}$, we normalize them to the weighting factor of the primary ions and invert them to get the relative transmission factors for the mass range of monomer, dimer, and trimer of the used perfluorinated acid with respect to the mass range of the primary ions.

The relative uncertainty was estimated via the $95 \%$ confidence bonds of the regression analysis. Additional uncertainty for the relative transmission factors comes from possible fragmentation of larger clusters to primary or monomer ions. However, as fragmentation to primary ions implicates that their signal cannot be fully depleted, and during the strongest heating the primary ion level goes, e.g., below 4 and $5 \%$ of the original level for heptanoic and nonanoic acid, respectively, we use $5 \%$ as upper limit for fragmen- tation to primary ions. For fragmentation to monomer ions, we compare the highest dimer signal to the monomer signal at the same point of time (the right peak of the monomers can be excluded for this, as no dimer contains $\mathrm{NO}_{3}^{-}$and thus cannot fragment to this peak). We find minimum monomer to dimer ratios of 12 and $20 \%$ for heptanoic and nonanoic acid, respectively. These ratios might go down even further for stronger heating, so we have added an additional uncertainty for primary ion signals of $5 \%$ and for all the others of $15 \%$. As the relative transmission of the primary ions was set to 1 , their uncertainty was added in quadrature to the one of all other compounds involved. Time resolution of the data affects the uncertainty originating from the regression analysis; we chose a $3 \mathrm{~s}$ resolution for all compounds, which leads to relatively small error bars. As the monomer peaks of all involved compounds spread over a certain $m / z$ range and the transmission efficiency may change over this span, summing up the three monomer peaks introduces additional uncertainty. Note that the ratios of primary ions, monomers, dimers and trimers to each other should vary ideally significantly over the duration of the experiment to obtain reliable results with this method, otherwise close to linearly dependent equations cause a large scatter of the results. At this point also the advantage of the heating method over the injection method becomes obvious as the first one produces smooth increases and decreases of perfluorinated acids and so much more data points for the statistical analysis.

\section{Results}

We have obtained two transmission curves for the HR-DMA method and one for the depletion method, which are shown in Fig. 5. The curves were obtained by applying least-squares fits to a 2-fold Gaussian distribution for the APi-, CI-mode and depletion data. To ensure reasonably good representation of the measured data, we had to set lower limits for the two parameters in the denominator of the exponent ( $200 \mathrm{for}$ the CI-mode and depletion method and 350 and 500 for the APi-mode fit). The overall transmission for the APi mode is in the range of $1 \%$ with a steep increase from $\mathrm{m} / \mathrm{z} 80 \mathrm{Th}$ $\left(\mathrm{Br}^{-}\right)$to $m / z 127 \mathrm{Th}\left(\mathrm{I}^{-}\right)$and a shallow decrease at higher $\mathrm{m} / \mathrm{z}$ ranges above around $700 \mathrm{Th}$. The shape of the transmission curve is in agreement with earlier estimates for an APi-TOF using a similar method by Ehn et al. (2011); however, in our study we found around transmission levels a factor of 2 higher than Ehn et al. (2011). The data points of the APi setup are systematically higher than for the CI setup, with a larger difference for smaller masses. This difference is due to a combined effect of the CI ion source as well as the discussed voltage step of $-500 \mathrm{~V}$ in front of the ion source. The result is a stronger suppression of lighter ions in the CI setup and a shift of the maximum of the curve towards higher masses. Furthermore, the difference in transmission between $\mathrm{Br}^{-}$and $\mathrm{I}^{-}$is much smaller for the CI setup. Note that io- 

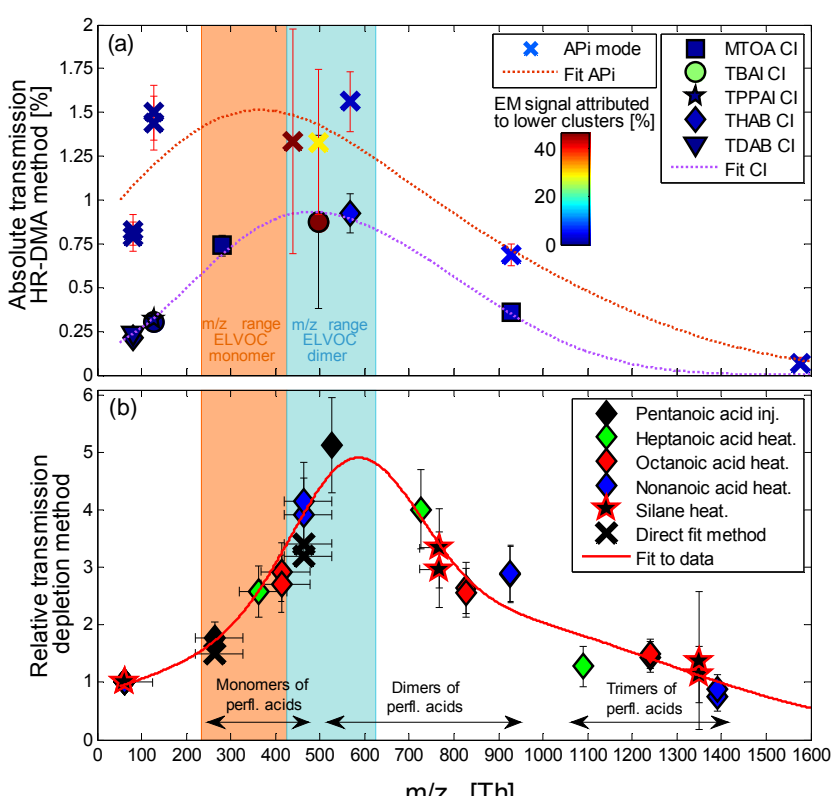

Figure 5. (a) Absolute transmission data obtained with the HRDMA method for APi only (crosses) and CI mode. Each data point is the ratio of TOF signal to corrected EM signal of a certain cluster. Iodide containing dimers fragment significantly to $\mathrm{I}^{-}$, which enhances uncertainty. (b) Transmission curve obtained by depletion method with included mass discrimination effects of the CI source. The data points obtained with the direct fit method represent lower limits of relative transmission. A least-squares fit to a 2-fold Gaussian distribution was applied to the data (direct fit data was not used for this fit). The transmission curve obtained by the depletion method changes significantly over the $m / z$ range of ELVOC molecules, indicated by orange and blue shaded areas for ELVOC monomer and dimer, respectively.

dide dimers show a high degree of fragmentation indicated by color code in Fig. 5a, whereas bromide dimers fragment very little. Therefore, bromide salts together with the ionic liquid MTOA work best for this method. Error bars in Fig. 5a represent results of Gaussian error propagation of the above mentioned uncertainties.

The results for the relative transmission efficiency estimated with the depletion method are shown in Fig. 5b. The agreement between the individual perfluorinated acids and the silane compound is remarkably good. There is a clear maximum of transmission efficiency in the range around $600 \mathrm{Th}$. Especially the $m / z$ range from 235 to $625 \mathrm{Th}$ shows a steep increase in transmission efficiency. This $m / z$ range corresponds to the ELVOC monomer and dimer range as indicated by light orange and blue color in Fig. 5 and is thus of great importance for ELVOC quantification. The direct fit method was applied to some of the data obtained during depletion experiments. It is a linear fit of product ions vs. reacted primary ions (as used in PTR-MS) during periods of time, where mostly monomers were present. These data points (black crosses in Fig. 5b) can be seen as a lower limit for the relative transmission. The above mentioned fit to the 2-fold Gaussian distribution of the results of the regression analysis was used as transmission curve of the instrument and to correct data sets from other experiments obtained under similar conditions. Horizontal error bars in Fig. 5b indicate the spread of the primary ions, as well as the monomers of the perfluorinated acids and the silane compound along the $m / z$ axis. All monomers of perfluorinated acids were set to the $m / z$ value of the deprotonated monomer peak (middle monomer peak, see Fig. 3). Vertical error bars originate from the regression analysis as described above. As the monomer peaks of all involved compounds spread over a certain $\mathrm{m} / \mathrm{z}$ range and the transmission efficiency changes over this span, summing up the three monomer peaks introduces additional uncertainty. However, as the left monomer peak of the perfluorinated acids has a lower transmission efficiency than the middle peak and the right monomer peak a higher one, this effect tends to cancel out. We tested this for all used compounds by correcting monomer peaks individually for transmission and found an effect ranging from $2 \%$ for perfluorinated pentanoic acid to $10 \%$ for the silane compound. These uncertainties were added in quadrature to the ones of the regression analysis and affect only monomers but not dimers and trimers.

If we use the results of the regression analysis for the individual acids and correct the corresponding time traces in Fig. 4 for transmission, their sum should yield a constant level over the duration of the experiment. This is the case for both acids shown in Fig. 4 (light blue line). The constant signal confirms our method and represents now the constant ion production in the $\mathrm{CI}$ source.

The shape and the position of maximum transmission differ from the two curves obtained by the HR-DMA method. These differences may be attributed to mass discrimination effects of the CI source because the HR-DMA method does, in principle, not account for this. The CI setup shown in Fig. $1 \mathrm{~b}$ tried to simulate these effects, but for the price of introducing an additional potential step of $-500 \mathrm{~V}$, which also may change the shape of the transmission curve. The HR-DMA method is a valid and established method for estimating the transmission efficiency of an APi-TOF. However, for a CI-APi-TOF the depletion method produces more reliable results as the CI source is included in the characterization as well. This method shows two main advantages: The first is that the instrument is used in its standard operation mode with the exact same settings as during sulfuric acid or ELVOC measurements, so no additional corrections have to be applied in order to obtain the transmission efficiency. The second main advantage is that no knowledge of the amounts of perfluorinated acids that enter the instrument is required for estimating the relative transmission efficiency. We just have to add sufficient amounts to deplete the primary ions significantly. This results in a simple and inexpensive measurement setup. 


\section{Conclusion}

We have presented an easy-to-use and straight-forward way of estimating the mass-dependent transmission efficiency of a nitrate-based CI-APi-TOF via the depletion of primary ions. This method is in use already for PTR-MS, but to our knowledge has not been applied to a nitrate-based CIAPi-TOF before. The additional difficulty compared to PTRMS is a strong clustering of the tested substances, which is treated with statistical analysis of the measurement data. We used different perfluorinated acids and one silane compound to estimate relative transmission factors over the $m / z$ range from around 250 to $1400 \mathrm{Th}$. The results for all compounds are very consistent and show a steady increase in transmission efficiency from the $\mathrm{m} / \mathrm{z}$ region of the nitrate primary ions up to about $500 \mathrm{Th}$ by a factor of $\sim 5$. A second method, which estimates the transmission efficiency of the APi-TOF alone without the CI source by use of an HR-DMA and an electrometer, shows a differently shaped transmission curve with a transmission efficiency of $\sim 1.5 \%$ in the $\mathrm{m} / \mathrm{z}$ region from 127 to $568 \mathrm{Th}$. We thus conclude that there most probably exist non-negligible mass discrimination effects of the CI source. These effects have to be taken into account for the quantification of ELVOCs and other compounds. As the transmission efficiency strongly depends on the chosen settings of the instrument, our transmission estimation cannot be generalized to other instruments. Ehn et al. (2014) found similar calibration coefficients for sulfuric acid and perfluorinated heptanoic acid, indicating similar transmission efficiencies for both $\mathrm{m} / \mathrm{z}$ ranges. However, a detailed transmission curve cannot be obtained by a standard gravimetrical calibration with one compound, so the method presented in this study can further enhance the accuracy of quantification for ELVOCs and other molecules. The depletion method has the advantage of a simple setup, as no need for quantification of the measured compounds exists and the instrument is used in its normal operation mode. We also want to point out that this method should, in principle, work as well for other chemical ionization schemes like acetate or iodide; however, different substances than the ones used in our study will have to be applied. 


\section{Appendix A}

Here we present the detailed settings of the CI-APi-TOF used in this study. The settings were applied for the depletion method as well as for HR-DMA experiments.

Table A1. Voltage and flow settings of the CI source. The basic design of the CI source is given in Kürten et al. (2011); however, the $\mathrm{HNO}_{3}$ and corona mixing flow were not fed into the source together with the $\mathrm{N}_{2}$ sheath flow, but directly over the corona needle. The electrostatic lens in front of the pinhole and the $\mathrm{N}_{2}$ buffer flow were omitted in our CI source. The corona needle voltage given below is reduced by a $500 \mathrm{M} \Omega$ resistor before being applied to the needle itself.

\begin{tabular}{lrlr}
\hline Gas flows in CI source & {$\left[\mathrm{L} \mathrm{min}^{-1}\right]$} & Voltages CI source & {$[\mathrm{V}]$} \\
\hline $\mathrm{N}_{2}$ sheath flow & 20 & Corona cylinder & -500 \\
Exhaust flow & 31.85 & Drift tube & -210 \\
$\mathrm{HNO}_{3}$ flow & 0.050 & Pinhole plate & 0 \\
Corona mixing flow & 1.5 & Corona needle & -6000 \\
Sample flow CI source & 9.5 & & \\
Sample flow TOF & 0.80 & & \\
\hline
\end{tabular}

Table A2. Voltages and frequencies in the APi section.

\begin{tabular}{lrlrll}
\hline \multicolumn{2}{l}{ SSQ low voltages [V] } & \multicolumn{2}{l}{ BSQ low voltages [V] } & Quadrupoles RF \\
\hline IMR & 0 & Q2 Front & 10 & SSQ Frequency & $620 \mathrm{kHz}$ \\
Nozzle & 10 & Q2 Back & 13.5 & SSQ Amplitude & $180 \mathrm{~V}$ \\
Q1 EP & 5 & Skimmer 2 & 16 & BSQ Frequency & $1.15 \mathrm{MHz}$ \\
Q1 Front & 8 & Reference (bias) & 17 & BSQ Amplitude & $100 \mathrm{~V}$ \\
Q1 Back & 8 & Ion-lens 2 & 135 & & \\
Lens skimmer & 8 & Detector flange & 39 & & \\
Skimmer & 8 & Deflector & 38.1 & & \\
\hline
\end{tabular}

Table A3. Voltages and pressures in the TOF section (operated in negative high sensitivity mode).

\begin{tabular}{lrlrll}
\hline \multicolumn{2}{l}{ Extraction pulser [V] } & \multicolumn{2}{l}{ TOF high voltages [V] } & \multicolumn{2}{l}{ TOF pressures [mbar] } \\
\hline TOF Pulse & 700 & Lens & 500 & Fore pressure & 3.52 \\
TOF Ref. & 42 & Drift & 3000 & SSQ & 2.29 \\
TOF Extr. 1 & 35 & RG & 649 & BSQ & $3.48 \times 10^{-2}$ \\
TOF Extr. 2 & 700 & RB & 700 & PB & $9.55 \times 10^{-5}$ \\
& & HM & 0 & TOF & $9.61 \times 10^{-7}$ \\
& & A & 2700 & & \\
& MCP & 2155 & & \\
\hline
\end{tabular}


Acknowledgements. We thank Jay Slowik for the fruitful discussion. We also thank the tofTools team for providing tools for mass spectrometry analysis. This work was funded by: EC Seventh Framework Programme (Marie Curie Initial Training Network, MC-ITN “CLOUD-TRAIN" no. 316662), German Federal Ministry of Education and Research (project no. 01LK1222A), the University of Innsbruck Research Grant for Young Scientists and the Austrian Science Fund, FWF (grant no. P27295-N20).

Edited by: F. Stroh

\section{References}

Chernushevich, I. V., Loboda, A. V., and Thomson, B. A.: An introduction to quadrupole-time-of-flight mass spectrometry, J. Mass Spectrom., 36, 849-865, 2001.

Edwards, G. D., Cantrell, C. A., Stephens, S., Brian, H., Goyea, O., Shetter, R. E., Mauldin III, R. L., Kosciuch, E., Tanner, D. J. and Eisele, F. L.: Chemical Ionization Mass Spectrometer Instrument for the Measurement of Tropospheric $\mathrm{HO}_{2}$ and $\mathrm{RO}_{2}$, Anal. Chem., 75, 5317-5327, 2003.

Ehn, M., Junninen, H., Schobesberger, S., Manninen, H. E., Franchin, A., Sipilä, M., Petäjä, T., Kerminen, V.-M., Tammet, H., Mirme, A., Mirme, S., Hõrrak, U., Kulmala, M., and Worsnop D. R.: An Instrumental Comparison of Mobility and Mass Measurements of Atmospheric Small Ions, Aerosol Sci. Tech., 45, 522-532, 2011.

Ehn, M., Thornton, J., Kleist, E., Sipilae, M., Junninen, H., Pullinen, I., Springer, M., Rubach, F., Tillmann, R., Lee, B., LopezHilfiker, F., Andres, S., Acir, I.-H., Rissanen, M., Jokinen, T., Schobesberger, S., Kangasluoma, J., Kontkanen, J., Nieminen, T., Kurten, T., Nielsen, L. B., Jorgensen, S., Kjaergaard, H., Canagaratna, M., Dal Maso, M., Berndt, T., Petaejae, T., Wahner, A., Kerminen, V.-M., Kulmala, M., Worsnop, D. R., Wildt, J., and Mentel, T. F.: A large source of low-volatility secondary organic aerosol, Nature, 506, 476-479, 2014.

Eisele, F. L. and Tanner, D. J.: Ion-assisted tropospheric OH measurements, J. Geophys. Res., 96, 9295-9308, 1991.

Eisele, F. L. and Tanner, D. J.: Measurement of the gas phase concentration of $\mathrm{H}_{2} \mathrm{SO}_{4}$ and methane sulfonic acid and estimates of $\mathrm{H}_{2} \mathrm{SO}_{4}$ production and loss in the atmosphere, J. Geophys. Res., 98, 9001-9010, 1993.

Fernández de la Mora, J. F. and Kozlowski, J.: Hand-held differential mobility analyzers of high resolution for $1-30 \mathrm{~nm}$ particles: Design and fabrication considerations, J. Aerosol. Sci., 57, 4553, 2013.

Gerlich, D.: Inhomogeneous RF fields: a versatile tool for the study of processes with slow ions, in: Advances in Chemical Physics: State-Selected and State-To-State Ion-Molecule Reaction Dynamics, Part 1, Experiment, edited by: Ng, C.-Y. and Baer, M., John Wiley \& Sons, New York, USA, 82, 1-176, 1992.

Gilmore, I. S. and Seah, M. P.: Ion detection efficiency in SIMS: Dependencies on energy, mass and composition for microchannel plates used in mass spectrometry, Int. J. Mass. Spectrom., 202, 217-229, 2000.
Graus, M., Müller, M., and Hansel, A.: High resolution PTR-TOF: quantification and formula confirmation of VOC in real time, J. Am. Soc. Mass Spec., 21, 1037-1044, 2010.

Hansel, A., Jordan, A., Holzinger, R., Prazeller, P., Vogel, W., and Lindinger, W.: Proton transfer reaction mass spectrometry: online trace gas analysis at the ppb level, Int. J. Mass Spectrom., 149-150, 609-619, 1995.

Huey, L. G., David R. H., and Carleton J. H.: Reactions of $\mathrm{SF}_{6}^{-}$and $\mathrm{I}^{-}$with atmospheric trace gases, J. Phys. Chem. US, 99, 50015008, 1995.

Jokinen, T., Sipilä, M., Junninen, H., Ehn, M., Lönn, G., Hakala, J., Petäjä, T., Mauldin III, R. L., Kulmala, M., and Worsnop, D. R.: Atmospheric sulphuric acid and neutral cluster measurements using CI-APi-TOF, Atmos. Chem. Phys., 12, 4117-4125, doi:10.5194/acp-12-4117-2012, 2012.

Junninen, H., Ehn, M., Petäjä, T., Luosujärvi, L., Kotiaho, T., Kostiainen, R., Rohner, U., Gonin, M., Fuhrer, K., Kulmala, M., and Worsnop, D. R.: A high-resolution mass spectrometer to measure atmospheric ion composition, Atmos. Meas. Tech., 3, 10391053, doi:10.5194/amt-3-1039-2010, 2010.

Kürten, A., Rondo, L., Ehrhart, S., and Curtius, J.: Performance of a corona ion source for measurement of sulfuric acid by chemical ionization mass spectrometry, Atmos. Meas. Tech., 4, 437-443, doi:10.5194/amt-4-437-2011, 2011.

Kürten, A., Rondo, L., Ehrhart, S., and Curtius, J.: Calibration of a chemical ionization mass spectrometer for the measurement of gaseous sulfuric acid, J. Phys. Chem. A, 116, 6375-6386, 2012.

Kürten, A., Jokinen, T., Simon, M., Sipilä, M., Sarnela, N., Junninen, H., Adamov, A., Almeida, J., Amorim, A., Bianchi, F., Breitenlechner, M., Dommen, J., Donahue, N. M., Duplissy, J., Ehrhart, S., Flagan, R.C., Franchin, A., Hakala, J., Hansel, A., Heinritzi, M., Hutterli, M., Kangasluoma, J., Kirkby, J., Laaksonen, A., Lehtipalo, K., Leiminger, M., Makhmutov, V., Mathot, S., Onnela, A., Petäjä, T., Praplan, A. P., Riccobono, F., Rissanen, M. P., Rondo, L., Schobesberger, S., Seinfeld, J. H., Steiner, G., Tomé, A., Tröstl, J, Winkler, P. M., Williamson, C., Wimmer, D., Ye, P., Baltensperger, U., Carslaw, K. S., Kulmala, M., Worsnop, D. R., and Curtius, J.: Neutral molecular cluster formation of sulfuric acid-dimethylamine observed in real time under atmospheric conditions, P. Natl. Acad. Sci. USA, 111, 1501915024, 2014.

Muntean, F.: Transmission study for rf-only quadrupoles by computer simulation, Int. J. Mass Spectrom., 151, 197-206, 1995.

Müller, M., Mikoviny, T., and Wisthaler, A.: Detector aging induced mass discrimination and non-linearity effects in PTR-ToF-MS, Int. J. Mass. Spectrom., 365, 93-97, 2014.

Steinbacher, M., Dommen, J., Ammann, C., Spirig, C., Neftel, A., and Prevot, A. S. H.: Performance characteristics of a protontransfer-reaction mass spectrometer (PTR-MS) derived from laboratory and field measurements, Int. J. Mass. Spectrom., 239, 117-128, 2004.

Steiner, G., Attoui, M., Wimmer, D., and Reischl, G. P.: A medium flow, high-resolution Vienna DMA running in recirculating mode, Aerosol Sci. Tech., 44, 308-315, 2010.

Tanner, D. J. and Eisele, F. L.: Present OH measurement limits and associated uncertainties, J. Geophys. Res., 100, 2883-2892, 1995. 